\title{
Design for reflection
}

\author{
Sebastiano Bagnara ${ }^{\mathrm{a}}$ and Simone Pozzi ${ }^{\mathrm{a}}$ \\ ${ }^{a}$ Faculty of Architecture, University of Sassari-Alghero, Palazzo del Pou Salit - Piazza Duomo 6, 07041 Alghero \\ (SS), Italy \\ ${ }^{\mathrm{b}}$ Deep Blue srl, Piazza Buenos Aires 20, 00198 Rome, Italy
}

\begin{abstract}
Since a few years, a number of academic papers have been proposing to shift from user-centered design to humancentered (or person) design. In this contribution, we discuss as the common tread underlying these works the idea that design should also address the reflective part of our human experience, and not only aim to maximize the experiential aspects. Our review is complemented with examples derived from the internet world and from ICT consumer products. The main research areas we see as promising for the approach of "design for reflection" are: design for pauses, design for detachment, design for serendipity.
\end{abstract}

Keywords: optimal flow, usability, user experience, reflection, emotional design

\section{Introduction}

In one of the scenes of "The Social Network" (on the story of Facebook's founder), we see a young software coder, completely immersed in his programming task. He is absorbed by the computer screen, with an almost physical connection through the headset cable. Two other characters are on stage, with one of the two telling the second not to bother the programmer because "He's wired in".

This scene is a nice and clear representation of one the most popular notions in the Human-Computer Interaction (HCI) field, that is the notion of the $o p$ timal flow. The term has been initially introduced by Csikszentmihaly [6], and then made widely popular by the works of Don Norman [15], to describe the state of full engagement that we sometimes experience while interacting with technologies. Our actions stay on the flow, neither too slow, nor too quick, and so is the task demand, which remains challenging without becoming too hard and frustrating. According to Norman, when we experience the optimal flow our attention stays focused and we remain completely immerged in our tasks. The external world and its other stimuli disappear and we live only for what we are doing at the present moment (i.e. our task). The optimal flow is an intense, pleasing and productive state, one that suits both the workplace and also the free time.
Designing for the optimal flow has almost become a goal in itself, one of the primary goals to pursue in HCI. An immersive experience is often considered an attribute of successful design products, with the user so connected to the product that $\mathrm{s} / \mathrm{h}$ e desires to interact with it as long as possible, with no interruptions. Yet, the popularity of the notion seems to be diminishing. If we search "optimal flow" in the books published in the last 20 years (by means of Google Books Ngram), there is a peak of citations around the mid of the Nineties, and then a slow, but continuous, descent till today.

Many researchers and practitioners start proposing design goals and frameworks that do not fit well with the optimal flow, and sometimes are even in open contradiction with it. For instance, Danzico [7] speaks of the importance of pauses and transition moments, while Fullerton [10] advocates the need of designing for solitude. These are just two instances, but they are, in our opinion, clear indications that some HCI researchers and practitioners have moved their focus from immersive interactions to the design of moments of reflection, to what we may name the design for reflection.

In the rest of this contribution, we will discuss a strand of Human-Computer Interaction approaches concerned with the idea that design should foster reflective thinking. 


\section{Some (Tacit) assumptions about the user}

The way the user is conceived in the design for the optimal flow has some peculiarities that deserve a full analysis. First of all, her/his well-being passes through a complete coupling with a technological tool. It is as if we were tacitly assuming that a rewarding experience happens whenever the whole person is reduced to one (or few) aspects, those that can be "wired in" a technological tool, those that can be fulfilled by performing a task at our maximum potential.

This is more the description of a workaholic (or of a hacker), someone who is fully motivated by her/his work, who does not experience fatigue nor boredom, and stays absorbed in her/his task.

We are probably rendering an oversimplified description of what the optimal flow proponents consider as being their users. Still, few in this area have ever questioned the possibility that a radical interpretation of the optimal flow might lead to a simplistic model of users. Among them, Don Norman has complemented the idea of optimal flow with the one of emotional design, to address also other aspects of human life [16]. Most of the practitioners seem instead to assume that good designs remove all the interaction obstacles, smooth the human-computer interaction to the point where users can engage in the optimal flow. Traditional usability approaches aim for maximum efficiency and efficacy, user experience approaches include aesthetic aspects and amusement, but all of them do not really question the key design objective: to optimize the human-machine fit, or to maximize the user performance by minimizing her/his cognitive load.

\section{The risks of optimal flow}

The aim of this contribution is not to question the relevance of the optimal flow notion for successful design. Many technological tools are today designed with the goal of supporting the optimal flow and they indeed succeed in doing that. For our pleasure and benefit. Our aim is to discuss how there is more than optimal flow to design for and to show some of the drawbacks of such a conception.

Just like any other design approach, the optimal flow implies design choices and the primacy of some design directions over others. This section will review some of the tensions that these design choices cannot resolve, or have exacerbated. We will briefly outline only some of the most relevant tensions, without any pretense of completeness, or depth of analysis.

\subsection{Everything that matters is happening today}

The mission of Google (the company that more represents the last ten years of technological developments) is to organize the world information and make it easily, universally and instantaneously available and accessible. This mission is particularly relevant in the age of information deluge, where we are constantly running the risk of information overload and we need to keep scanning the available information to make sure we are not missing the important pieces.

However, there is a drawback to this overabundance. Most of the information we gather and process will become old in a very brief period of time, no matter what its value is, it will decay very quickly in a short time. For instance, we keep learning new software and internet applications, only to discover that the new release is out. We learn new skills and interaction modes, but we cannot rely on them. What we have learned yesterday is already old, and will not serve to any purpose tomorrow. Our skills do not add up, we have no time (nor incentive) to master and deeply appropriate them. Instead they require constant upgrade and revision.

The pace of innovation is so high that our memory runs the risk of becoming a burden. We need to forget in order to effectively learn, in order not to be constrained by the old habits. We are facing the paradoxical situation where we need to voluntarily "erase" skills and competences on which we invested time and efforts [2]. We need to forget in order to achieve the optimal flow with the new technologies, in order to be able to quickly learn new skills.

Our memory lives in a fast moving present, where technologies do not give us any indication to consciously choose what to remember and what to forget [3].

\subsection{Is multi-tasking making us stupid?}

The overabundance of information stimulates skills such as multi-tasking, or the capability to cope with continuous interruptions. We need to be able to quickly move from one information flow to the next one, so quickly that we are almost paying attention to two or three flows at the same time. It is the skill of parallel thinking, that becomes unrenounceable to engage in multiple tasks, to carry out several differ- 
ent projects with different audiences and teams. Not a new skill, but one on which we are more and more relying to cope with the current technological scenario.

The drawback in this case is the loss of reflective thinking, of the capability to engage in complex speculations, to stay focused on only one specific topic. In one famous essay, Carr [5] describes a scenario in which we all engage only in superficial reading, far from the concentration required to read an old-fashioned book, much more similar to "a form of skimming activity, hopping from one source to another".

Carr mentions some selected studies to support his argument, but admits that there is no scientific longterm proof of such changes. Still, for the sake's of this contribution, it should not appear too much controversial to state that if we design technologies to support "skimming behaviors", we are likely to penalize more linear ways of reading and to offer cues (to skim and hop) that are likely to disrupt the oldfashioned reading rhythm.

\subsection{Too many weak ties}

Internet has made the creation of virtual community an easy endeavor. New communities keep being born and new social networks aggregate large numbers of users.

But most of these communities are short lived and tend to dissolve in few years. These communities are based on the idea that being connected is what constitutes a community, without any other form of stronger bonds. There is little (or no) emotional elaboration in such communities, most of the communication acts actually communicate little else than the contact itself. The like/dislike function on Facebook is a good example of the primacy of the contact function on the communication contents. Likewise, we may mention the short format (on average) of Facebook posts. Few persons are going to invest time and resources in long posts, when they know their post will disappear from their friends' main page after few hours.

For these reasons, community members find it easy to leave a community. They have not made any real investment in it, so the opt-out costs are low. And they can afford to move to another community.

The consequences on the psychological life in those communities is too complex to be discussed here, but it is worth mentioning how such dynamics also impact on the self perception of our bodies. According to Sherry Turkle [20], being immerged in a continuous flow of interaction with new technologies causes our bodies to almost disappear. The optimal flow is so engaging that we forget our bodily matter, we are wholly absorbed by the computer interface to the point that we forget that our self also resides in our body, and not only in our computer-mediated interactions.

\section{Designing for reflection}

The discipline of HCI has played a major role in shaping the web world, by making it more userfriendly, engaging and accessible. It suffices to mention the popularity and wide impact of Jakob Nielsen's usability heuristics [14]. However, the right time might have arrived to critically revise if HCI is properly equipped to support the whole variety of human activities, including those that required focused attention and reflection. It is probably less a matter of having the right methods and techniques available, than a need to critically revise some assumptions and move in directions not yet fully explored till now.

The rest of our contribution will present three design directions that may be worthwhile pursuing:

- design for pauses,

- design for detachment,

- design for serendipity.

The underlying idea is that the best way to support reflective thinking is to design spaces and moments where reflection can take place without being constrained by the rhythm of some external "flow" (of experience, of events, of inputs, etc...). These spaces should offer the user the opportunity to "leave the flow" of continuous interaction with technology.

\subsection{Design for pauses}

The dialectic interplay of experiential cognition and reflective cognition is a hallmark of human cognition. The issue has been discussed by Norman in [15], but also in the seminal book by Winograd and Flores [21].

Winograd and Flores analyzed the concept of breakdown, that is moments where the humantechnology interaction does not flow smoothly and the user has to stop and engage in a problem solving activity. Breakdowns were traditionally seen as something to be avoided, being disruptive of the current activity. The authors suggest instead that these moments also play a fundamental role in human cognition, by making it possible for technology to 
emerge as a thing in itself and not only as a transparent medium. The tool becomes impossible to use during a breakdown, thus bringing the user attention on the tool itself and fostering an enhanced understanding of the tool characteristics and of its principles of functioning.

A first design direction might be to explore the means by which "breakdown moments" can be recreated, of course without any actual breakdown. In other words, tools should be able to attract the user's attention on their characteristics before breakdowns occur, they should be able to activate the enhanced understanding triggered by breakdowns as a design feature, not as a side-effect of bad functioning.

Under this respect, a desirable characteristic of breakdowns that should be recreated is when the user distances her/himself from the flow of activity, looking at it from a different perspective and restructuring her/his perception of the normal tool functioning. Breakdowns open a space suitable for reasoning, a space that often leads to innovation, as well summarized in Petrovski's motto "Form follows failure" [17]. When confronted with a breakdown, humans try to devise a second (often better) solution. A reflective tool should be able to trigger such an innovation mechanism without relying on actual failure.

Following this line of reasoning, pauses should not be regarded as something to be avoided, but as moments where reflective reasoning can more effectively take place. They make it possible for the user to rest and recover from the fatigue of a continuous interaction but, more importantly, they also offer the possibility to shift our mode of behavior from the experiential one to the reflective mode [7].

One example is the debriefing carried out by aircraft pilots before and after a flight, or in flight to prepare for high peak and high demand flight phases (e.g. landing). It is a moment of pause, where the crew prepares itself for the performance moment, when distance is sought from the flow of events to mark a change of rhythm and intensity. Or it may be a transition out of the performance phase, where a collective reflection is carried out to analyze what went well and what could be improved. Debriefings serve a variety of purposes (depending on the domain and on the moment when they are carried out), but it is safe to say that they are characterized by a problem setting attitude, as opposed to the problem solving one. Problems are re-defined during debriefings, which are moments dedicated to reframing the usual into a new form. New problems may also emerge, ones that were not identified before.
Designing for pauses may also imply designing for solitude [10]. Not solitude as a dysfunctional separation from the others (like Robert De Niro in Taxi Driver), but solitude as a solid barrier against distraction [4] (like Martin Heidegger walking in the woods).

Solitude may be a pre-condition to avoid continuous interruptions, or the constant contact without content that sometimes characterizes web communities. To enable reflective thinking, pauses may not be enough and physical detachment may also be necessary.

\subsection{Design for detachment}

There is another way of leaving the flow of activity to reach a space for reflection. It is the one enabled by the change of perspective, by detachment in order to see the problem (or the activity) under a different light.

The example that is currently most prominent is the growing field of visual analytics, that the visual representation of huge data sets [19]. The information deluge has created the need for tools that help graphically summarize complex data sets, in order to enable analysis and comparison without getting lost in infinite minute details.

In this case, turning information into knowledge requires to step outside the flow of details, to focus on the level of emerging patterns and find the correct interpretation. Knowledge cannot be achieved without detachment, without distancing the interpretation from single events. Visual analytics demonstrate how experiential and reflective design can be fruitfully combined. Software tools like Gapminder make it a matter of optimal flow the elaboration of data, but only in order to then trigger reflection on the emerging patterns.

Another example is the Change Laboratory method, developed by Engeström and colleagues [9]. The Change Laboratory invites workers to use categories derived from the Scandinavian Activity Theory [13] (i.e. subject, object, tool, rule, division of labor, and community) to reframe everyday working life in abstract categories. The goal is solve workplace issues by using theory as a form of detachment. For instance, participants are invited to frame problems as tensions between the basic 6 categories: tools may be unfit for the current goals, current rules may be ambiguous or far from reality, and so forth. These tensions cannot be solved if faced directly, while participants find it easier to deal with 
them by addressing from the meta-level of theory. On this level, most tensions can be solved by referring to common organizational goals.

Videogames also provide some examples of detachment to support reflective thinking. Most Nintendo Wii games have been designed to maximize the optimal flow experience. However, other games demand users to increase their self-perception, for instance to control their body posture while playing a sport (e.g. yoga practice, or skiing). Microsoft has also delivered a console that can be controlled by body movements and gestures, named Kinect. In this case some games are based on the idea of acting (or mimesis), for instance by playing the part of an animal, moving one's own body and see the animal's body move.

In all these games, the user can rely on the game consoles to operate a form of detachment, to see one's self as in a mirror, thus making it possible to engage in reflective thinking and restructure her/his behavior.

\subsection{Design for serendipity}

Danzico [8] notes how the strict compliance to design guidelines as "less is more" may bring about an unintended drawback: "People may be less exposed to chance or less inclined to try new things; behavior may be planned such that there are no discoveries or surprises. Technology may be increasing the opportunity for specificity, but is it decreasing our chances for serendipity?". By leaving out what is not directly related to the end goal, designers are also diminishing the space for fortuitous discoveries. The tourist armed with her/his smart phone will go straight to her/his hotel, but will never stumble upon something unexpected.

The use of serendipity to support creative thinking has deep roots. During the Enlightenment, many authors used to take notes of noticeable quotes, seminal ideas, reflections, or book excerpts, by means of a commonplace book. John Locke had even devised a tagging method to make it possible to find notes at a later time, while at the same time leaving them unsorted and mixed. By reading the commonplace book after some time, the author was likely to see new connections among all these unsorted thoughts, by reading one note after the other, in an order different than the one in which these had been written.

The commonplace book was a loosely organized collector of thoughts, designed to keep together notes but at the same time leaving space for serendipitous connections. By reading the commonplace book, the authors could reflect on their own past thoughts, and also profit from the mix between order and disorder to trigger new ideas.

As with the other directions we have discussed, serendipity rests on the optimal balance between two polarities: randomness and order. It is not all or nothing, experiential or reflective, or total randomness. We maintain that HCI should pursue this balance by considering both instances, by supporting the users on both dimensions, without reducing human experience to just one dimension. Web services like Dopplr or Hitotoki combine both goal directed features (like restaurant recommendations, or hints about a nw city) with other ones that support serendipity. Hitotoki asks users to post online photographs of off-the-beaten-tracks places, to take photos while moving from one famous monument to the next one. Hitotoki aims to build maps of serendipity, maps that reflect the unexpected discoveries made by its users. It asks tourists to avoid being enmeshed in the optimal flow, in order to stay detached and find those details often overlooked.

\section{Concluding remarks}

In the recent years, there has been a flourishing body of academic works increasingly focused on the idea that user-centered design should become person (or human) centered, thus encompassing the person as a whole, not only as a user. This may relate to socio-cultural aspects $[11,12]$, to serendipity or moments of pause $[7,8]$, or even to advocating for the design of solitude [10]. In a similar line, Norman has discussed the advantages of emotional approaches to design [16], encompassing aspects like physical sensations, or values and meanings we attach to products.

The underlying idea of all these works is that by reducing design to just the useful bits (i.e. those aimed at efficiency and efficacy, at achieving goals), we cut out aspects that are the hallmark of being humans. When design focuses on what is strictly needed for one task, then the chances of serendipitous encounters, or of explorative behaviors, are very limited.

In our opinion, such emerging approach can be named "design for reflection". We maintain that serendipity, pauses, a certain degree of detachment, are all relevant features to engage in reflective thinking. Examples of the need for reflective thinking can be found in the current patterns of web use [1], where users are alternating a lean forward attitude (typical 
of the internet of the nineties), with the lean back attitude that marks less interactive media, like TV. This attitude change is coherent with what Premsky [18] calls the homo sapiens digital, who shifts away from compulsive interaction modalities to a more balanced use of technology.

In order to orient Human-Computer Interaction towards reflective thinking, we maintain that we should critically revise our implicit assumptions about our users, to address the needs and motivations of the whole person. We should shift from goaloriented users, to persons who pursue both experiences and reflections. The risk we are currently running is to break down the unity of a person into task-oriented fragments.

\section{References}

[1] C. Anderson and M. Wolff, The Web Is Dead. Long Live the Internet, Wired, (2010).

[2] S. Bagnara, R. Montanari, and S. Pozzi, Designing Organisational Oblivion, in: Industrial Engineering and Ergonomics. Visions, Concepts, Methods and Tools, C. Schlick, ed. Springer Verlag, Berlin, Germany. 2009,pp. 233-242.

[3] L.J. Bannon, Forgetting as a feature, not a bug: the duality of memory and implications for ubiquitous computing, CoDesign, 2(1) (2006), 3-15.

[4] M.G. Berman, J. Jonides, and S. Kaplan, The Cognitive Benefits of Interacting with Nature, Psychological Science, 19(2) (2008), 1207-12.

[5] N. Carr, Is Google making us stupid?, The Atlantic, (JulyAugust) (2008).
[6] M. Csikszentmihalyi, Flow: The Psychology of Optimal Experience, Harper \& Row, New York, NY, 1990

[7] L. Danzico, Adding By Leaving Out: The Power of the Pause, interactions, 17(4) (2010), 55-57.

[8] L. Danzico, The Design of Serendipity Is Not by Chance, interactions, 17(5) (2010), 16-18.

[9] Y. Engeström, J. Virkkunen, M. Helle, J. Pihlaja, and R. Poikela, Change laboratory as a tool for transforming work, Lifelong Learning in Europe, 1(2) (1996), 10-17.

[10] B. Fullerton, Designing for Solitude, interactions, 17(6) (2010), 6-9.

[11] R. Harper, T. Rodden, Y. Rogers, and A. Sellen, eds, Being Human: Human-Computer Interaction in the year 2020, Microsoft Research Ltd, Cambridge, UK, 2008.

[12] J. Kolko, On Experiences, People, and Technology, interactions, 17(6) (2010), 80.

[13] B.A. Nardi, Context and consciousness : activity theory and human-computer interaction, MIT Press, Cambridge, MA, 1996.

[14] J. Nielsen, Designing Web Usability : The Practice of Simplicity, New Riders Publishing, Indianapolis, IN, 1999.

[15] D.A. Norman, Things that make us smart : defending human attributes in the age of the machine, Addison-Wesley Pub. Co., Reading, MA, 1993.

[16] D.A. Norman, Emotional design : why we love (or hate) everyday things, Basic Books, New York, NY, 2004.

[17] H. Petroski, The Evolution of Useful Things, Vintage Books, New York, NY, 1994.

[18] M. Premsky, H. Sapiens Digital: From Digital Immigrants and Digital Natives to Digital Wisdom, Innovate, Feb-Mar (2009).

[19] The Economist, Data, data everywhere, (2010).

[20] S. Turkle, Alone together: Why we expect more from technology and less from each other, Basic Books, New York, NY, 2011.

[21] T. Winograd and F. Flores, Understanding Computers and Cognition: A New Foundation for Design, Addison Wesley Publishing Company, Reading, MA., 1986. 